NASA/TM-2004-213106

\title{
Cross-Stream PIV Measurements of Jets With Internal Lobed Mixers
}

James Bridges and Mark P. Wernet

Glenn Research Center, Cleveland, Ohio 
Since its founding, NASA has been dedicated to the advancement of aeronautics and space science. The NASA Scientific and Technical Information (STI) Program Office plays a key part in helping NASA maintain this important role.

The NASA STI Program Office is operated by Langley Research Center, the Lead Center for NASA's scientific and technical information. The NASA STI Program Office provides access to the NASA STI Database, the largest collection of aeronautical and space science STI in the world. The Program Office is also NASA's institutional mechanism for disseminating the results of its research and development activities. These results are published by NASA in the NASA STI Report Series, which includes the following report types:

- TECHNICAL PUBLICATION. Reports of completed research or a major significant phase of research that present the results of NASA programs and include extensive data or theoretical analysis. Includes compilations of significant scientific and technical data and information deemed to be of continuing reference value. NASA's counterpart of peerreviewed formal professional papers but has less stringent limitations on manuscript length and extent of graphic presentations.

- TECHNICAL MEMORANDUM. Scientific and technical findings that are preliminary or of specialized interest, e.g., quick release reports, working papers, and bibliographies that contain minimal annotation. Does not contain extensive analysis.

- CONTRACTOR REPORT. Scientific and technical findings by NASA-sponsored contractors and grantees.
- CONFERENCE PUBLICATION. Collected papers from scientific and technical conferences, symposia, seminars, or other meetings sponsored or cosponsored by NASA.

- SPECIAL PUBLICATION. Scientific, technical, or historical information from NASA programs, projects, and missions, often concerned with subjects having substantial public interest.

- TECHNICAL TRANSLATION. Englishlanguage translations of foreign scientific and technical material pertinent to NASA's mission.

Specialized services that complement the STI Program Office's diverse offerings include creating custom thesauri, building customized databases, organizing and publishing research results ... even providing videos.

For more information about the NASA STI Program Office, see the following:

- Access the NASA STI Program Home Page at http://www.sti.nasa.gov

- E-mail your question via the Internet to help@sti.nasa.gov

- Fax your question to the NASA Access Help Desk at 301-621-0134

- Telephone the NASA Access Help Desk at 301-621-0390

- Write to:

NASA Access Help Desk

NASA Center for AeroSpace Information 7121 Standard Drive

Hanover, MD 21076 
NASA/TM-2004-213106

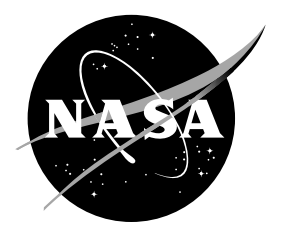

\section{Cross-Stream PIV Measurements of Jets With Internal Lobed Mixers}

James Bridges and Mark P. Wernet

Glenn Research Center, Cleveland, Ohio

Prepared for the

Tenth Aeroacoustics Conference

cosponsored by the American Institute of Aeronautics and Astronautics and the Confederation of European Aerospace Societites

Manchester, United Kingdom, May 10-12, 2004

National Aeronautics and

Space Administration

Glenn Research Center 
Available from

NASA Center for Aerospace Information 7121 Standard Drive

Hanover, MD 21076
National Technical Information Service 5285 Port Royal Road Springfield, VA 22100

Available electronically at http://gltrs.grc.nasa.gov 


\title{
Cross-Stream PIV Measurements of Jets with Internal Lobed Mixers
}

\author{
James Bridges and Mark P. Wernet \\ National Aeronautics and Space Administration \\ Glenn Research Center \\ Cleveland, Ohio 44135
}

\begin{abstract}
Summary
With emphasis being placed on enhanced mixing of jet plumes for noise reduction and on predictions of jet noise based upon turbulent kinetic energy, unsteady measurements of jet plumes are a very important part of jet noise studies. Given that hot flows are of most practical interest, optical techniques such as Particle Image Velocimetry (PIV) are applicable. When the flow has strong azimuthal features, such as those generated by chevrons or lobed mixers, traditional PIV, which aligns the measurement plane parallel to the dominant flow direction is very inefficient, requiring many planes of data to be acquired and stacked up to produce the desired flow cross-sections. This paper presents PIV data acquired in a plane normal to the jet axis, directly measuring the cross-stream gradients and features of an internally mixed nozzle operating at aircraft engine flow conditions. These nozzle systems included variations in lobed mixer penetration, lobe count, lobe scalloping, and nozzle length. Several cases validating the accuracy of the PIV data are examined along with examples of its use in answering questions about the jet noise generation processes in these nozzles. Of most interest is the relationship of low frequency aft-directed noise with turbulence kinetic energy and mean velocity.
\end{abstract}

\section{Nomenclature}

D long duct nozzle diameter

$\mathrm{D}_{\mathrm{e}} \quad$ equivalent thrust jet diameter

$\mathrm{D}_{\mathrm{m}} \quad$ mixed jet diameter

$\mathrm{U}_{\mathrm{m}} \quad$ mixed jet velocity

$\beta \quad$ area ratio

$\delta \quad$ density ratio

$\lambda \quad$ velocity ratio

ASD Acoustic Source Density

LDV Laser Doppler Velocimetry

PIV Particle Image Velocimetry

TKE Turbulent Kinetic Energy

Conf Confluent (axisymmetric) splitter

12CL 12-lobe, Low penetration mixer

12UM 12-lobe, Medium penetration mixer

12UH 12-lobe, High penetration mixer

L0 Long nozzle

L1 Medium nozzle

L2 Short nozzle 


\section{Introduction}

Projections show that jet noise will continue to be a constraining factor in aircraft noise, hence there is a continued need to understand, predict, and reduce jet noise. All three of these aspects of jet noise research benefit from knowledge of the turbulent velocity field of jets, particularly when the jet is hot and high speed. Particle Image Velocimetry (PIV) has emerged as a strong technology for measuring flows of all temperature and speeds with only minimal constraints. One of those constraints has been the inability to acquire data in cross-stream planes where the flow gradients of most interest occur. This is especially important in jets with forced mixers or chevrons.

In a recent test cosponsored by NASA's Quiet Aircraft Technology program and Allison Advanced Development Corporation, a portion of the test was dedicated to implementing cross-stream stereo PIV for the study of internally mixed long-duct nozzles. This test was conducted in the Nozzle Acoustic Test Rig (NATR) at the NASA Glenn Research Center using model hardware first tested during a 1996 test documented in reference 1.The purpose of the 2003 test was to answer questions concerning certain acoustic anomalies observed during the 1996 test. (Preliminary analysis of the far-field acoustics will be presented in a paper in this conference by Tester and Fisher. ${ }^{2}$ ) To address these questions using standard PIV with planes aligned with the dominant flow direction would have required tens of planes of data be acquired at each axial location to map out the lobed structure of the flow with the likelihood that the data in the planes would not have fit together well enough to provide the level of detail desired. Cross-stream PIV provided this data in a few axial planes, greatly increasing the number of configurations that could be investigated in an equivalent amount of facility test time.

3-D PIV has been used in previous flow field studies. ${ }^{3,4,5}$ From a measurement accuracy standpoint the optimal configuration is to have the light sheet plane aligned with the dominant flow direction to capture the particle displacements at the two instants that the light sheet is pulsed. Placing the light sheet in the cross-flow orientation (light sheet perpendicular to the dominant flow direction) typically results in lower accuracy velocity measurements since the allowable range of the particle displacements is limited by the light sheet thickness. However, the efficiency gained in acquiring cross-sectional planes drove the decision to implement the cross-flow measurement configuration.

In this paper some results of validation work are presented, while the remainder of the paper gives short summaries of applications of the PIV data to the questions addressed by the test.

\section{Experimental Setup}

\section{Jet Rig}

The experiments were conducted on the dual flow jet rig in the Nozzle Acoustic Test Rig (NATR) at the NASA Glenn Research Center's AeroAcoustic Propulsion Laboratory. This dual flow engine simulator provides exhaust gases at pressure and temperature conditions typical of modern turbofan engines with mass flows up to $65 \mathrm{~kg} / \mathrm{second}$. The NATR is a $1.35 \mathrm{~m}$ freejet in which the jet rig sits, providing a moving freestream around the jet plume at speeds up to $\mathrm{M}=0.35$.

\section{Model Hardware and Test Matrix}

The test hardware simulates a BPR 4, internally mixed nozzle system with jet nozzle diameter of 184 $\mathrm{mm}$. Three lengths of nozzle were tested as shown in figure 1(a), having lengths of 1.5, 1.14, and 0.76 nozzle diameters beyond the mixer plane and designated L0, L1, and L2 respectively. An axisymmetric splitter served as the baseline for the variations in mixer geometry. Also of interest to this paper are three 12-lobed mixers, dubbed 12CL, 12UM, and 12UH with penetrations $0.5,0.7,0.88$ of the mixer plane annulus respectively, measured peak to peak. These are pictured in figure 1(b) along with the 
(a)

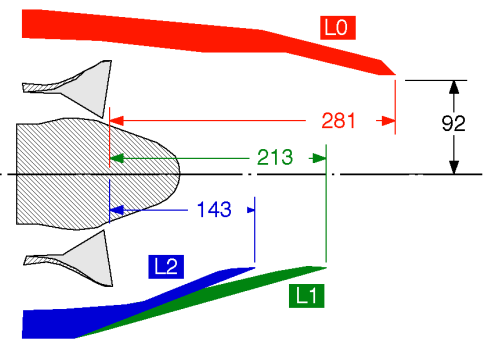

(b)

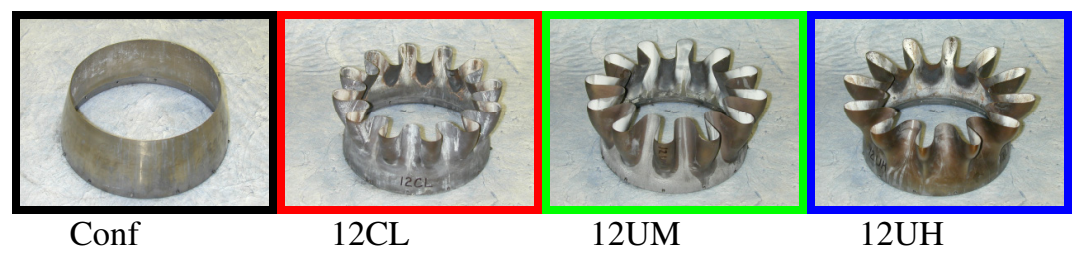

Figure 1.-Nozzle hardware components. (a) nozzles of different lengths relative to mixer; (b) 12-lobed and axisymmetric mixers.

confluent (axisymmetric) splitter which served as the baseline configuration. Data are presented here include four flow conditions listed in table 1. Two realistic jet flow conditions, setpoints 0112 and 0312 , included a simulated forward flight speed of $M=0.2$. In order to replicate data acquired using LDV in the previous test of the hardware, a setpoint 0101 was also tested, differing from setpoint 0112 primarily in the temperature of the fan stream. Setpoint 5000, with both streams set equal to produce a single cold static jet, was run on the confluent splitter geometry.

Table 1.-Definition of setpoint flow conditions reported

\begin{tabular}{|c|c|c|c|c|c|}
\hline Setpt ID & $\mathrm{NPR}_{\text {core }}$ & $\mathrm{NPR}_{\text {fan }}$ & $\mathrm{NTR}_{\text {core }}$ & $\mathrm{NTR}_{\text {fan }}$ & $\mathrm{M}_{\mathrm{FJ}}$ \\
\hline 0112 & 1.39 & 1.44 & 2.799 & 1.196 & 0.20 \\
\hline 0312 & 1.74 & 1.82 & 3.337 & 1.196 & 0.20 \\
\hline 0101 & 1.39 & 1.43 & 2.34 & 1 & 0.10 \\
\hline 5000 & 1.435 & 1.435 & 1 & 1 & 0.00 \\
\hline
\end{tabular}

\section{PIV and Data Processing}

To obtain velocity fields in jet cross-sections, the PIV system had to be configured counter to best practice, which would have the largest component of velocity contained within the light sheet. However, using stereo PIV, the cross-stream configuration is feasible. The error in the in-plane velocity estimates is inversely proportional to the particle displacement between exposures. The out-of-plane velocity estimate is obtained from the difference of the two in-plane velocity estimates and scales as the inverse tangent of the coupling half angle ${ }^{6}$. When the stereo PIV system is configured such that the largest component of the jet flow is oriented out of the plane of the light sheet, as in figure 2 the stereo cameras' oblique view of the illuminated plane results in apparent in-plane displacements that are larger than the actual out-of-plane displacements. Hence, the measured in-plane displacements, which are used to compute the axial velocity component, can be relatively large, even for small particle displacements. 


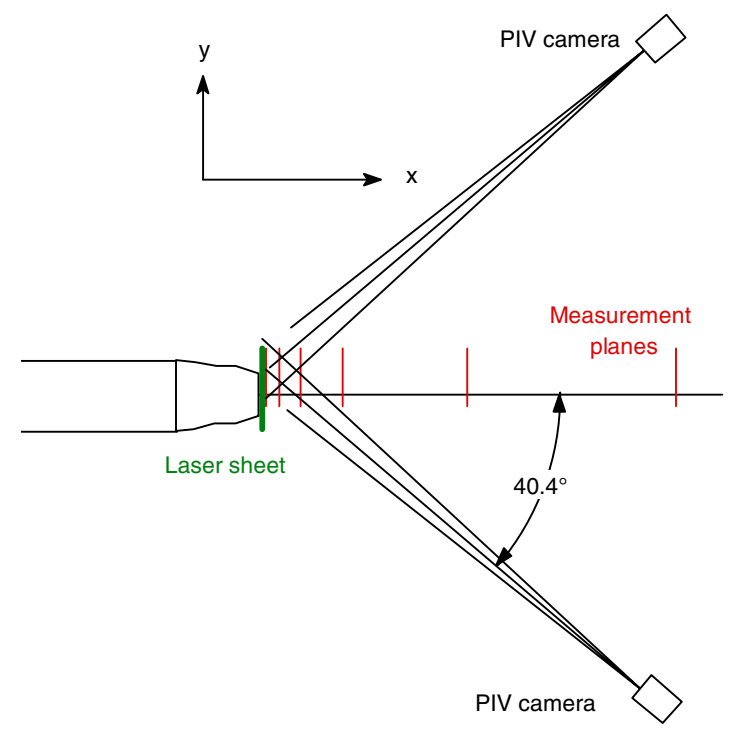

Figure 2.-Cross-stream stereo PIV applied to jet flow.

The allowable particle displacements were maximized via the light sheet configuration. The most accurate cross-correlations are obtained when the same ensemble of particles are recorded on the first and second exposures. One approach is to offset the location of the light sheets in the primary flow direction, which works when the flow velocity is relatively uniform. If the flow has a wide dynamic range, this approach will not work. If the light sheets are made thicker and fully overlap, then a wide dynamic range of flows can be measured; however, the ensemble of particles recorded is not optimal. Many particles are recorded in the two illuminated planes that are not in common, hence, they do not actually contribute to the correlation process and instead add noise on the correlation plane. The configuration used in this work, which enabled a wide dynamic range of velocities to be measured and minimized the number of extraneous particles in the recorded images, used a thin sheet for the first laser pulse and a thick sheet for the second laser pulse. The first sheet was positioned at the upstream edge of the thicker second laser sheet. The ensemble of particles illuminated in the first laser sheet were then illuminated by the second laser sheet regardless of their velocity (there were no negative velocities in this experiment). Extensive checks of the method, such as comparisons with standard single cold jet flows and repeats of experiments validated the robustness of the procedure.

The PIV system was implemented using two $2 \mathrm{~K} \times 2 \mathrm{~K}$ pixel PIV acquisition cameras, connected to separate computers, synchronized to a $400 \mathrm{~mJ} /$ pulse dual head PIV Nd:YAG laser. The laser sheet was formed normal to the jet axis, producing a plane of illumination in the cross-section of the jet. The two cameras were set on either side of the jet at angles $40^{\circ}$ from the jet axis approximately $2.2 \mathrm{~m}$ away from their image planes. Cameras, computers, and laser were all mounted on a large axial traverse and the image acquisition process controlled by PIVACQ $^{7}$ software.

The cameras were set up to image a region roughly $150 \mathrm{~mm} \times 150 \mathrm{~mm}$. This region was offset towards a quadrant of the jet to measure as great a radial distance as possible while slightly overlapping the centerline. The stereo PIV system was calibrated using a $200 \mathrm{~mm}$ by $200 \mathrm{~mm}$ grid target, which was translated over 5 axial planes.

Figure 3(a) gives a spatially accurate picture of the planes of data that were acquired during the test. Twelve measurement planes were spaced at exponentially increasing intervals in the axial direction from $0.1 \mathrm{D}$ to $10 \mathrm{D}$ to provide fine details of the initial evolution of the shear layers from the mixer immediately downstream of the nozzle and to cover the potential core region.

The jet stream flows were seeded using $0.5 \mu \mathrm{m}$ alumina powder dispersed in the flow well upstream of the nozzle using an air-assisted atomizing nozzle to atomize the $\mathrm{pH}$ adjusted ethanol dispersion of 

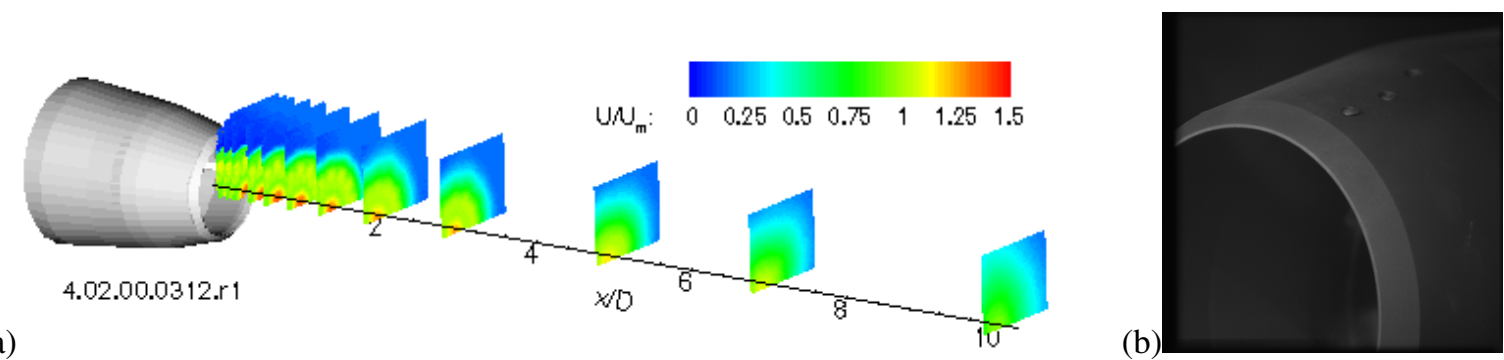

Figure 3.-(a) Orientation of data planes and nozzle system. (b) Background image from first plane showing location of nozzle in image.

alumina particles ${ }^{8}$. Two identical systems were used, one for the core flow and one for the fan flow. The ambient fluid was seeded with $0.2 \mu \mathrm{m}$ oil droplets produced by a commercial 'smoke' generator.

One problem degrades the velocity maps from the first few planes near the nozzle. Because of the viewing angle, the nozzle was in the background of the imaging plane for the planes from $0<\mathrm{x} / \mathrm{D}<1$ (see figure 3(b)) When the light sheet was within a few millimeters of the nozzle, light scattered from the ambient seed illuminated the nozzle and caused the nozzle lip to show strongly in the particle images. This reduced the signal to noise ratio in those regions of the image and sometimes showed up as erroneous features in the flow, particularly in the top dead center of the first few data planes where the nozzle images overlapped in the right and left camera views.

The processing of the two PIV views was done separately using PIVPROC, ${ }^{7}$ a NASA-produced PIV analysis software package. Multi-pass correlations were used, with the final pass having a spatial resolution of $16 \times 16$ pixels, reducing the $4 \mathrm{M}$ pixel images to two-dimensional displacement maps with 126 cells by 126 cells. Warping and merging of the two distorted velocity maps resulted in a common planar map of 103 by 139 three-component velocity cells, with a resolution of $1.06 \mathrm{~mm}$, or $0.005 \mathrm{D}$.

Individual vectors were qualified by three criteria. First, if the peak correlation of the image plane was not more than 1.5 times the nearest peak in the correlation plane, the velocity vector was marked invalid. Second, when combining the two oblique two-component vector maps from each camera view, if the common velocity component disagreed by more than 5 percent, the point was again marked as invalid. Finally, Chauvenet's criterion was applied to the ensemble of velocity vector components at each point in the measurement plane to reject outlier vectors before calculating the statistics for that point from the remaining valid points. Two hundred velocity maps were acquired for each location and used in computing turbulence statistics. The fraction of the 200 possible samples that were valid at a point was used to weigh the validity of higher order statistics at that point. Experience shows that as this fraction, called the 'quality', diminishes, higher order statistics become more questionable. Data at points with quality below 0.8 were not considered in the analysis and show up as blanks in the plots.

For some analysis the cross-sections were transformed into circular cylindrical coordinates and azimuthally averaged over an integral number of mixer lobes to compare against axisymmetric jets.

\section{Results}

\section{Validation studies}

Several efforts were made to directly validate the data from the cross-stream stereo PIV setup. First, data were acquired while both streams were operating at a cold $\mathrm{M}=0.7$ condition. Results are compared with published hotwire data ${ }^{9}$ for a single stream jet. In figure 4 , mean and rms axial velocity profiles acquired by the PIV system are shown at various axial locations, normalized by shear layer coordinates. The collapse of the data along the curves determined from hot wire work on dual flow jets ${ }^{10}$, gives confidence in the measurement technique. 

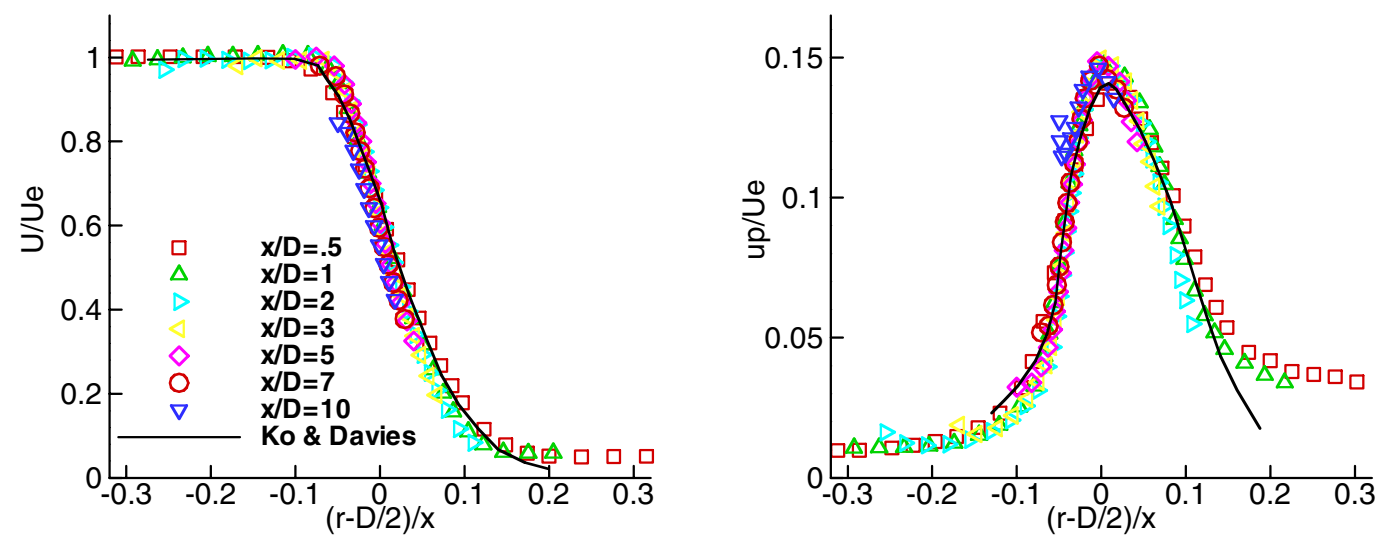

Figure 4.-Standard jet measurements obtained in $\mathrm{M}=0.7$ cold jet, achieved by running both streams at same condition with $M=0.05$ freestream velocity. (a) radial profiles of axial mean velocity at various axial stations, (b) radial profiles of axial turbulence intensity at various axial stations.
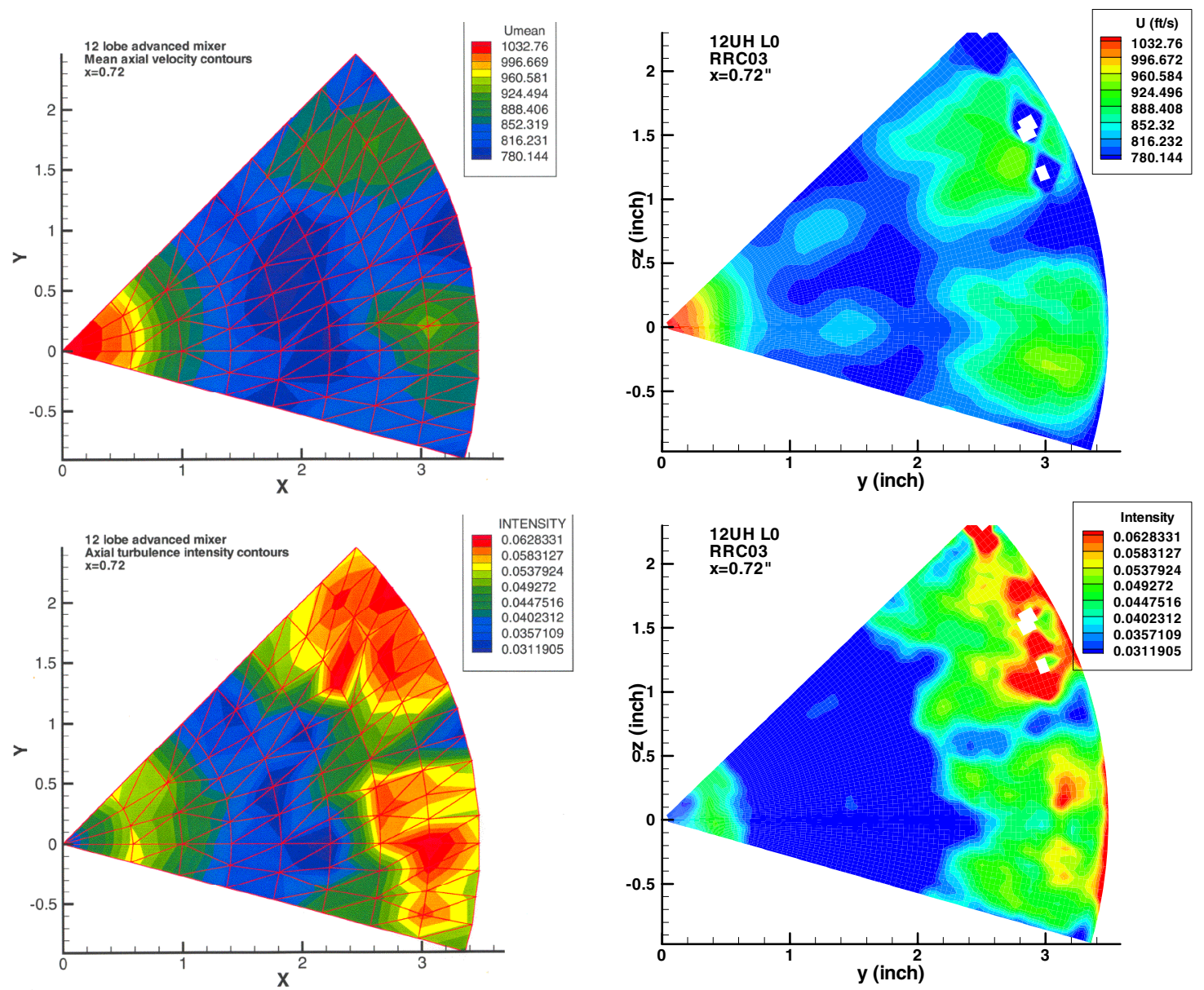

Figure 5.-Comparisons of LDV (left) and cross-stream PIV (right) measurements on high penetration (12UH) mixer with long (L0) nozzle at setpoint 0101.

Mean axial velocity (top), axial turbulence intensity (bottom). 
Second, a test point acquired in 1995 using LDV $^{11}$ was measured using PIV primarily to compare turbulence intensity maps. Plotting mean and RMS velocity on the same color contours as the printed contour maps of the LDV data acquired in 1995 (figure 5) shows agreement between the two measurements to within 3 percent and 10 percent of full-scale for mean and rms velocity respectively. Note that this is not only a validation of the PIV instrumentation but also of the repeatability of the entire test procedure since the two data sets were taken on different jet rigs.

Finally, the spatial resolution and temporal resolution of the processing was varied to assess the sensitivity of the turbulence statistics on these parameters. Factors of 2 variation in time between light sheet pulses and in spatial resolution of correlation volumes made little difference in the statistics. In fact, shortening the time delay between pulses by a factor of 5 only gave slightly different results, showing that the cross-stream PIV method is robust.

\section{Transonic Mixer Noise}

During earlier far-field acoustic tests it had been noted ${ }^{1}$ that there was a distinct hump in the spectra of these internally mixed nozzles when operated at setpoint 0312 , but not at the lower power condition, setpoint 0112. Interestingly, the hump appeared for short and long nozzles, but not for an intermediate length nozzle. Furthermore, the hump appeared at different frequencies for the short and long nozzles, differing roughly by a factor of 2 . This behavior was observed for several lobed mixers with different lobe counts and penetrations. The nozzle lengths and internal contours are given graphically in figure 1(a). Far-field spectra of the 12CL (low penetration) mixer with the three different length nozzles, shown in the figure, highlights the problem acoustically. At setpoint 0312, a spectral hump was observed at $3 \mathrm{kHz}$ for the long L0 nozzle, while it was observed at $6 \mathrm{kHz}$ for the short L2 nozzle and not at all for the medium L1 nozzle. This phenomenon was very repeatable, being present in the 1995 test data and in the 2003 data shown here.

Finding the source of this high frequency noise became one of the objectives of the 2003 test program. Several hypotheses were proposed for this source: a source internal to the rig, upstream of the mixer, a source downstream of the mixer but upstream of the nozzle, either produced by the internal shear layer, by unsteady separation on the mixer, or by shocks at mixer, or by impingement of the internal shear layer with the nozzle lip producing a ring tone. Several diagnostic tools were brought to bear on the issue, including cross-stream stereo PIV.

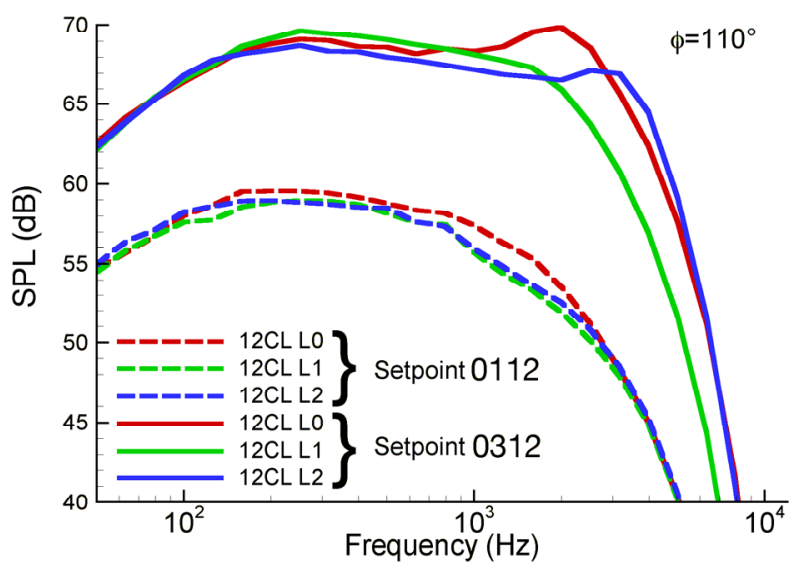

Figure 6.-One-third octave SPL at $110^{\circ}$, fullscale,

for low penetration (12CL) mixer in long (LO),

medium (L1), and short (L2) nozzles

at setpoints 0112 and 0312 . 


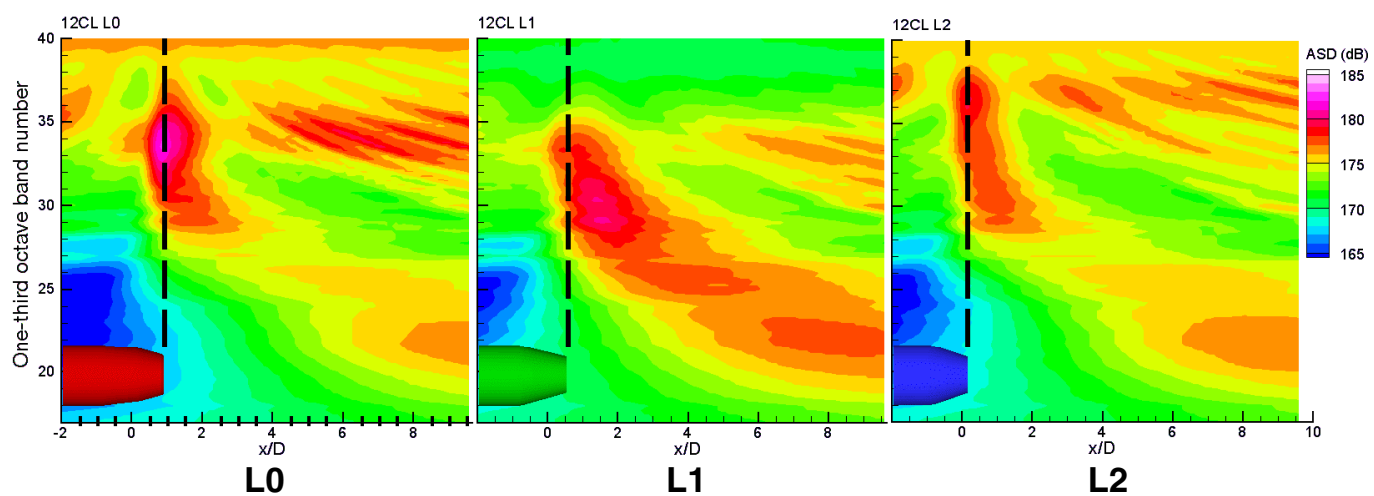

Figure 7.-Acoustic Source Density (ASD) for low penetration (12CL)mixer in long (L0), medium (L1), and short (L2) nozzles at setpoint 0312.

The most obvious way to find a noise source is with a phased array, in this case a nonlinearly spaced line array with 16 microphones, paralleling the jet axis some $20 \mathrm{D}$ off the jet centerline. ${ }^{12}$ The source distributions measured are given in figure 7 scaled in frequency to full-scale one-third octave bands to confirm the apparent location of the source. Some interpretation of phased array data is necessary. Note that while the axial scale is referenced to the static location of the long nozzle L0, the graphic of the nozzle and dashed line indicate the apparent location of the nozzle exit after convection by the surrounding freejet has been accounted for. Also understand that the apparent strong sources along bands at high frequencies downstream of $\mathrm{x} / \mathrm{D}=4$ are sidelobes of the main peak near the nozzle exit, not actual sources.

There was a clear difference between the acoustic source distributions of the medium length nozzle L1 and the shortest and longest nozzles, both of which show strong sources at the appropriate frequency very close to the nozzle. What was not clear from this picture was whether the noise was being generated inside the nozzles and releasing at the nozzle exit, or being generated at the exit or very shortly downstream. To look for an external source the flow field at the exit was examined to look for differences related to nozzle length in a non-monotonic fashion.

Figure 8 compares the mean and rms velocity fields near the exit of the three nozzles. In the plots of nozzles L0 and L1 one can see the artifacts from the light scattered from the nozzle in the particle images - the signal to noise of the image correlations in those regions of the particle images were sufficiently low that the data quality metric fell below the 0.8 threshold and the data was blanked out as unreliable. Using common sense and symmetry one can ignore these artifacts and analyze the data.

The mean axial velocity (figure 8(a)) clearly showed the high speed streaks associated with the lobes of the mixer and showed their spatial location relative to the nozzle shear layer. The shorter nozzles exhibited less mixing of these streaks as the nozzle exit was closer to the mixer. The plots of axial turbulence (figure 8(b)) gave a similar story, with monotonic progression of turbulence intensity with nozzle length in these high speed streaks.

The axial vortices created by the lobed mixer (figure 8(c)) also showed a monotonic increase in strength with reduced nozzle length, reflecting the reduced distance downstream of the mixer. The fancore shear layer was nowhere near the nozzle lip at the exit, so shear layer impingement seemed unlikely to be the source. In short, there was nothing that made the medium length nozzle flow field stand out as being different from both the short and long nozzles. Since no correlation was found between flow features at the nozzle exit and the prominent acoustic source in question, the PIV results strongly support the hypotheses of an internal noise source, with changes in internal nozzle contours being the reason for the medium length nozzle being different from both the short and long nozzles. 


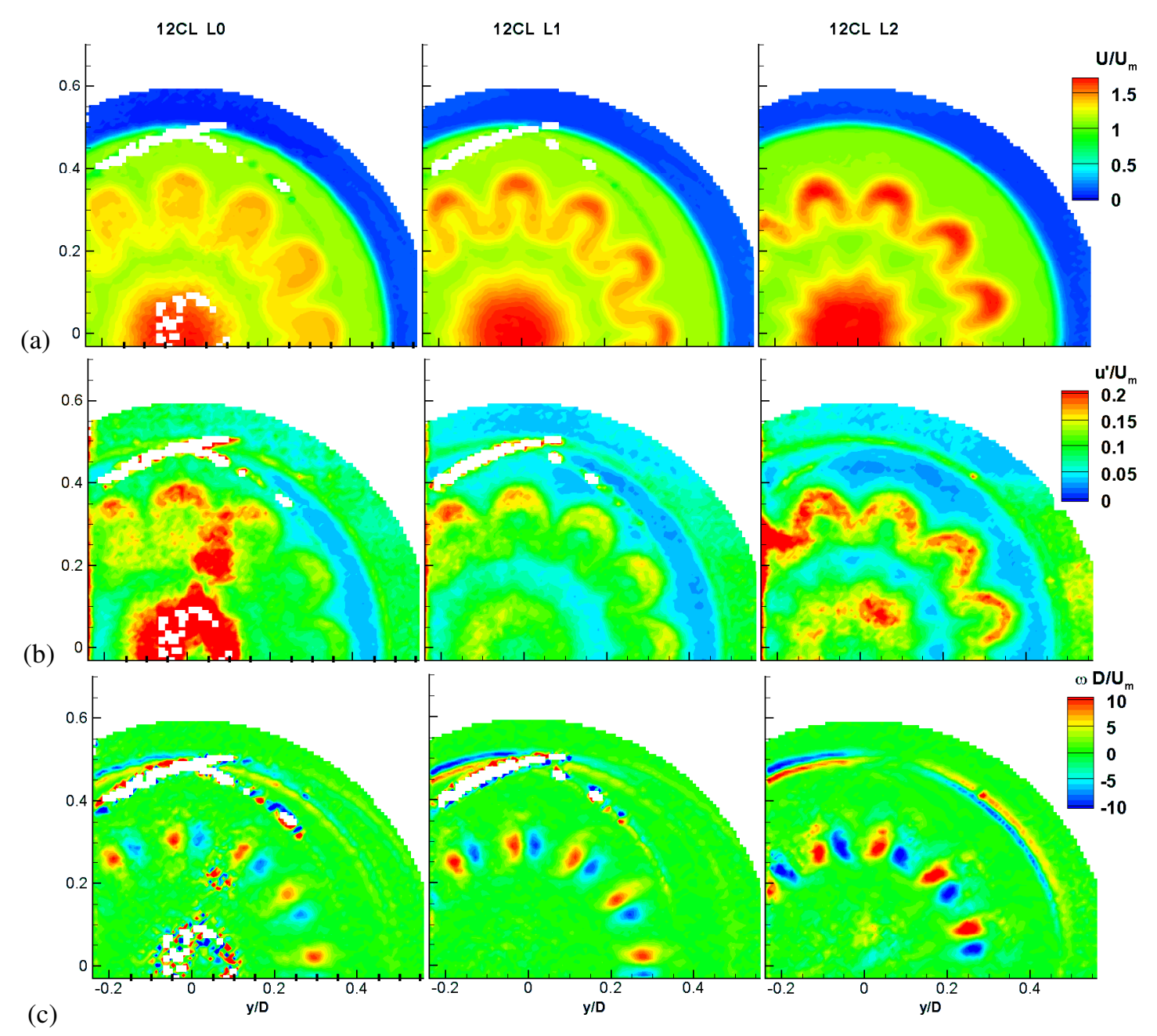

Figure 8.-Contours at axial station $\mathrm{x} / \mathrm{D}=0.2$ of (a) mean axial velocity, (b) rms axial velocity, and (c) axial vorticity for low penetration (12CL) mixer in long (L0), medium (L1), and short (L2) nozzles at setpoint 0312. Data was normalized by nozzle diameter $\mathrm{D}$ and mixed jet velocity $\mathrm{U}_{\mathrm{m}}$.

\section{Effect of Mixer Penetration}

As a general rule in jet noise, increasing mixing between low and high speed streams using forced mixers or chevrons results in a trade-off wherein increasing mixing reduces aft, low frequency noise, but increases broadside, high frequency noise. The internally mixed nozzles with lobed mixers tested here were no exception. Specifically, increasing the penetration of a lobed mixer produced more mixing and the aforementioned acoustic trade-off. Determining how to optimize this geometry, whether using an empirical model ${ }^{13}$ or an acoustic analogy, requires data about the turbulence field, either to validate assumptions in the model or to validate CFD predictions of the flow.

Results from three 12-lobe mixers with differing penetrations are presented here along with an axisymmetric confluent (Conf) splitter in a long nozzle (L0). The mixers are shown in figure 1 . The low penetration mixer (12CL) had slightly different lobe shapes from the medium (12UM) and high (12UH) penetration mixers. The far-field acoustic signature was summarized by one-third-octave spectra at $110^{\circ}$ and $150^{\circ}$, shown in figure 9 , scaled to a full-scale $1500 \mathrm{ft}$ level flyover. Data in this figure were acquired 
(a)

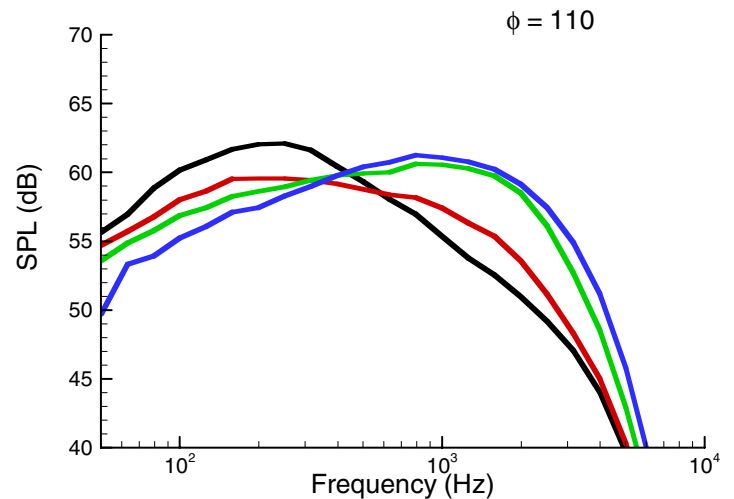

(b)

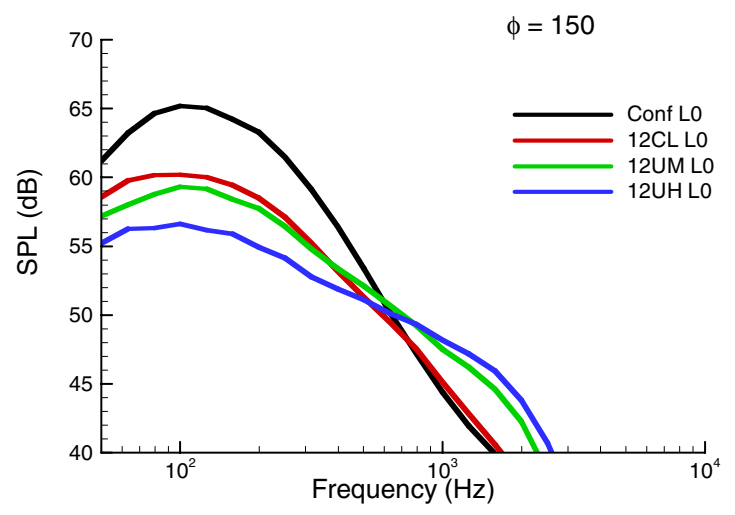

Figure 9.-1/3 octave spectra at (a) broadside and (b) aft angles for low (12CL), medium (12UM), and high (12UH) penetration mixers and axisymmetric (Conf) splitter in long (L0) nozzle at setpoint 0112. Data transformed to fullscale level flight on standard day.

at setpoint 0112 , but similar trends were found for setpoint 0312 with the addition of the high frequency source examined above. At broadside angles $\left(110^{\circ}\right)$ a high frequency lift was observed in the lobed mixers, being very strong in the medium (12UM) and high (12UH) penetration mixers. Conversely, all the lobed mixers showed over $5 \mathrm{~dB}$ suppression at low frequencies in the aft angle $\left(150^{\circ}\right)$, with the high penetration mixer reducing noise almost $10 \mathrm{~dB}$.

Phased array measurements produced axial distributions of sources at different frequencies (figure 10) for the different mixer configurations. Again, the sidelobes of the main peak at high frequencies must be ignored when interpreting these figures and the nozzle graphic should be used to locate the nozzle exit, accounting for the freestream advection of the apparent source location. The axial range of the plots were chosen to correspond to that measured using PIV and could be enlarged to fully capture the low frequency peak downstream of $\mathrm{x} / \mathrm{D}=10$.

Although the general trends of the acoustic source distributions (ASD) were not surprising, the extent to which the lobed mixers transfer acoustic power to a high frequencies near the nozzle was impressive. In particular, band number $25(3.2 \mathrm{kHz})$ showed a very strong shift in location, from $\mathrm{x} / \mathrm{D}>6$ to $\mathrm{x} / \mathrm{D}=2$, between the confluent and higher penetration mixers.

Although the detailed PIV data acquired in cross-sections, such as figure 8 , were very fascinating to examine, better quantitative analysis was made using azimuthal averages of the statistical fields. In particular, one can try different scaling parameters in an attempt to collapse the dual stream jet with single stream jets for jet noise modeling. ${ }^{13}$ One scaling approach is to geometrically scale the jet to that of a single-stream jet with equivalent thrust by rescaling the jet diameter and keeping the core velocity. The equivalent jet diameter $\mathrm{D}_{\mathrm{e}}$ is then

$$
\mathrm{D}_{\mathrm{e}}=\mathrm{D}(1+\lambda \beta), \quad \lambda=\frac{\mathrm{U}_{\text {fan }}}{\mathrm{U}_{\text {core }}}, \quad \beta=\frac{\mathrm{A}_{\text {fan }}}{\mathrm{A}_{\text {core }}},
$$

where $\mathrm{D}$ is the common flow nozzle diameter. This is intended to keep the main shear in the potential core constant and should give the best scaling for the potential core region. 


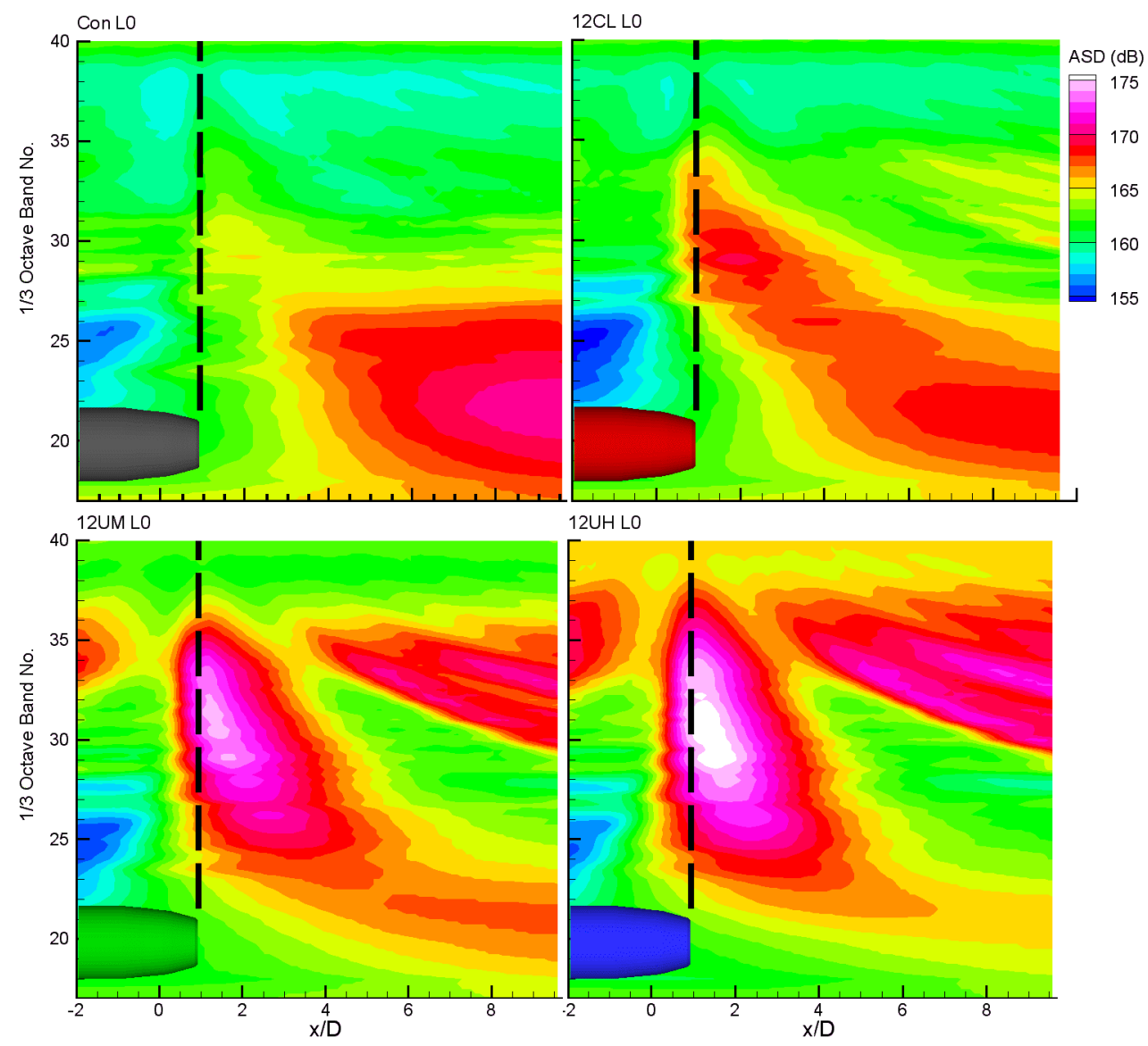

Figure 10.-Acoustic Source Distribution (ASD) for low (12CL), medium (12UM), and high (12UH) penetration mixers and axisymmetric (Conf) splitter in long (L0) nozzle at setpoint 0112. Data acquired using 16 microphone line array, imaged using conventional beamforming and transformed to fullscale third-octave bands.

Farther downstream a normalization producing equivalent mass and momentum was found to do a better job of scaling coaxial jet plumes. The mean velocity plumes have been found to achieve satisfactory collapse to that of a single-stream cold jet after a few diameters while the turbulence intensity was significantly reduced, from 15 to 10 percent. Called the mixed jet diameter $\mathrm{D}_{\mathrm{m}}$ and mixed velocity $\mathrm{U}_{\mathrm{m}}$ by Fisher, Preston, and Bryce ${ }^{14}$ the scaling parameters are given by

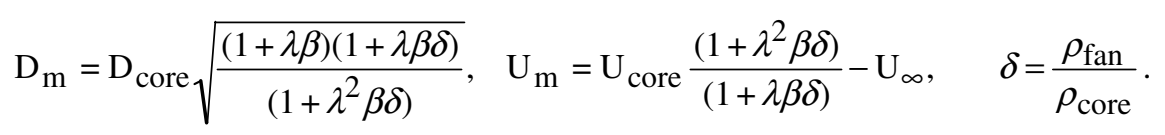

Note that equation (2) differs from the definition of reference 14 by the subtraction of the ambient (flight) speed $U_{\infty}$ from $U_{m}$. Because more configurations were acquired at setpoint 0312 , and because the normalized flow fields at setpoints 0112 and 0312 were very similar, data at setpoint 0312 are presented here. However, the results seem to be applicable to the setpoint 0112 case. In addition, one static, cold single-stream jet case (setpoint 5000, $\mathrm{M}=0.7$ ) is also presented for comparison. Here $\lambda=0.6, \beta=3$, $\delta=2.8$ for setpoint 0312 , producing $\mathrm{D}_{\mathrm{e}}=130 \mathrm{~mm}, \mathrm{D}_{\mathrm{m}} \sim \mathrm{D}=184 \mathrm{~mm}$, and $\mathrm{U}_{\mathrm{m}} \sim 290 \mathrm{~m} / \mathrm{s}$ for setpoint 0312 and $\mathrm{D}_{\mathrm{e}}=\mathrm{D}_{\mathrm{m}}=\mathrm{D}, \mathrm{U}_{\mathrm{m}} \sim 223 \mathrm{~m} / \mathrm{s}$ for the $\mathrm{M}=0.7$ static single-stream cold jet (setpoint 5000 ). 
Figure 11 shows the azimuthally averaged mean and RMS axial velocity fields normalized by $\mathrm{U}_{\mathrm{e}}$ and $D_{e}$ for the cold static single jet created by running statically with both streams at $M=0.7$, and for the axisymmetric splitter and three different mixers at the setpoint 0312 condition. When nondimensionalized by 'equivalent thrust' scalings, the nondimensionalized mean velocity fields for the coannular and singlestream jets look similar, but obviously the turbulence is much less in the case of the coannular nozzles.

When the plume data is normalized by mixed jet parameters $D_{m}$ and $U_{m}$, the mean velocity field was substantially different from a single-stream cold jet. The turbulence, thus normalized, was nearly the same for single-stream jets and for the dual flow jets, including the lobed mixers.

Comparing the turbulence distributions of figure 11 with the acoustic source distributions of figure 10 there was a good correspondence between the regions of high frequency noise generation near the nozzle and the levels of turbulence there. However, although the high penetration mixers had reduced low frequency noise, they had stronger turbulence at the downstream extent of the data, roughly 10 diameters. This is counter to expectations that the downstream 'end of potential core' region, where the axisymmetric and low penetration mixers showed more acoustic source strength, would have a correspondingly high turbulence level. Checks of other factors, such as turbulence anisotropy, did not satisfy the apparent paradox. Apparently the stronger low frequency noise at aft angles was associated with a mechanism not directly related to turbulent kinetic energy.

The fact that the relationship between turbulent kinetic energy and acoustic source broke down for low frequency, aft-directed sound should hardly be a surprise. This is precisely the region where the predictive codes based upon acoustic analogies show a strong dependence upon refraction, and where even at transonic conditions, instability waves can produce acoustic waves directly. These issues have led to the commonly held idea of a large-scale structure source. Both of refraction and instability waves are related to the gradients of mean velocity and hence, in terms of correlating flow features with the aft angle acoustics, the main correlating characteristic was the mean velocity.

For the purposes of modeling jet noise, the approach of Fisher ${ }^{13}$ proposes to rescale mean profiles to that of a single jet and to adjust source strength based upon turbulence intensity. The data of figure 11 and 12 can be used to guide the development of these models. But for addressing acoustic analogy prediction codes a more detailed investigation is required. PIV data can provide the data needed by many competing theories to quantify and validate their concepts.

Currently, there are several analytical approaches being considered to explain this phenomenon. Many focus on the growth and decay of instability waves which are dependent to first order upon the local mean velocity profile. PIV data can be used to provide these profiles, and can be used to validate these approaches. Papamoschou ${ }^{15}$ relates convective Mach numbers $\left(\mathbf{M}_{c}\right)$ of the shear layers within dual flow jets to acoustic source strength. In this method, local $M_{c}$ are determined for each shear layer by measuring
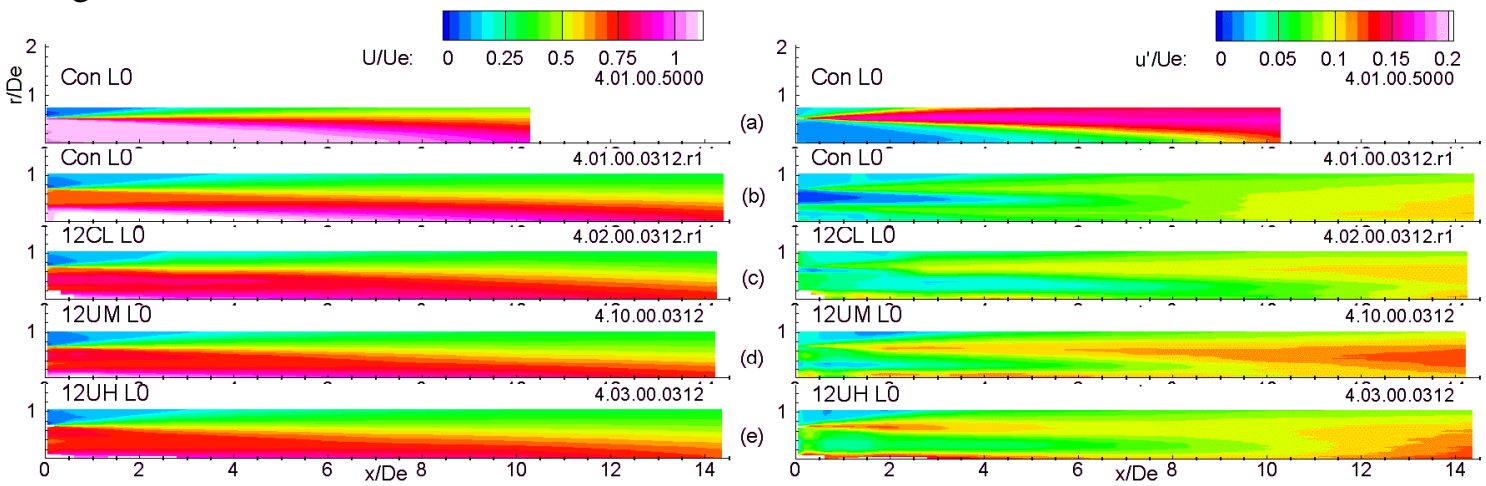

Figure 11.-Azimuthally averaged mean (left) and RMS (right) axial velocity fields, normalized by equivalent jet diameter $D_{e}$ and core velocity $U_{e}$, for (a) single-stream static jet at setpoint 5000, (b) axisymmetric splitter at setpoint 0312, and (c-e) three 12-lobe mixers with different penetrations on long (L0) nozzle at setpoint 0312. 


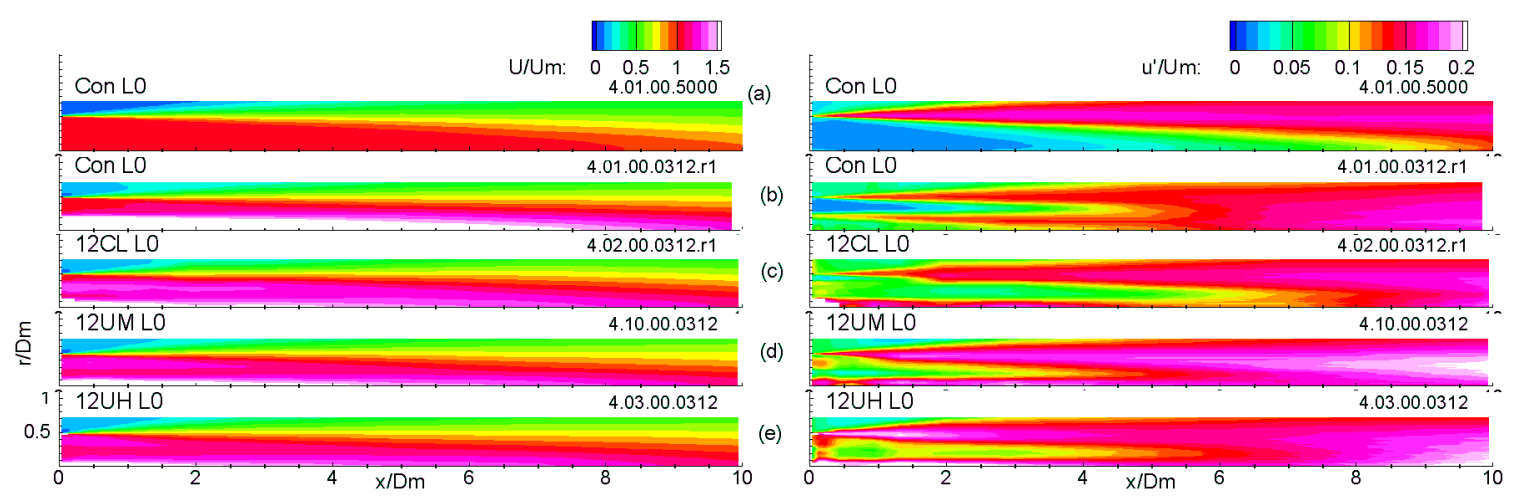

Figure 12.-Azimuthally averaged mean (left) and RMS (right) axial velocity fields, normalized by mixed jet diameter $\mathrm{D}_{\mathrm{m}}$ and mixed jet velocity $\mathrm{U}_{\mathrm{m}}$.

Same geometry and setpoint labels as figure 11.
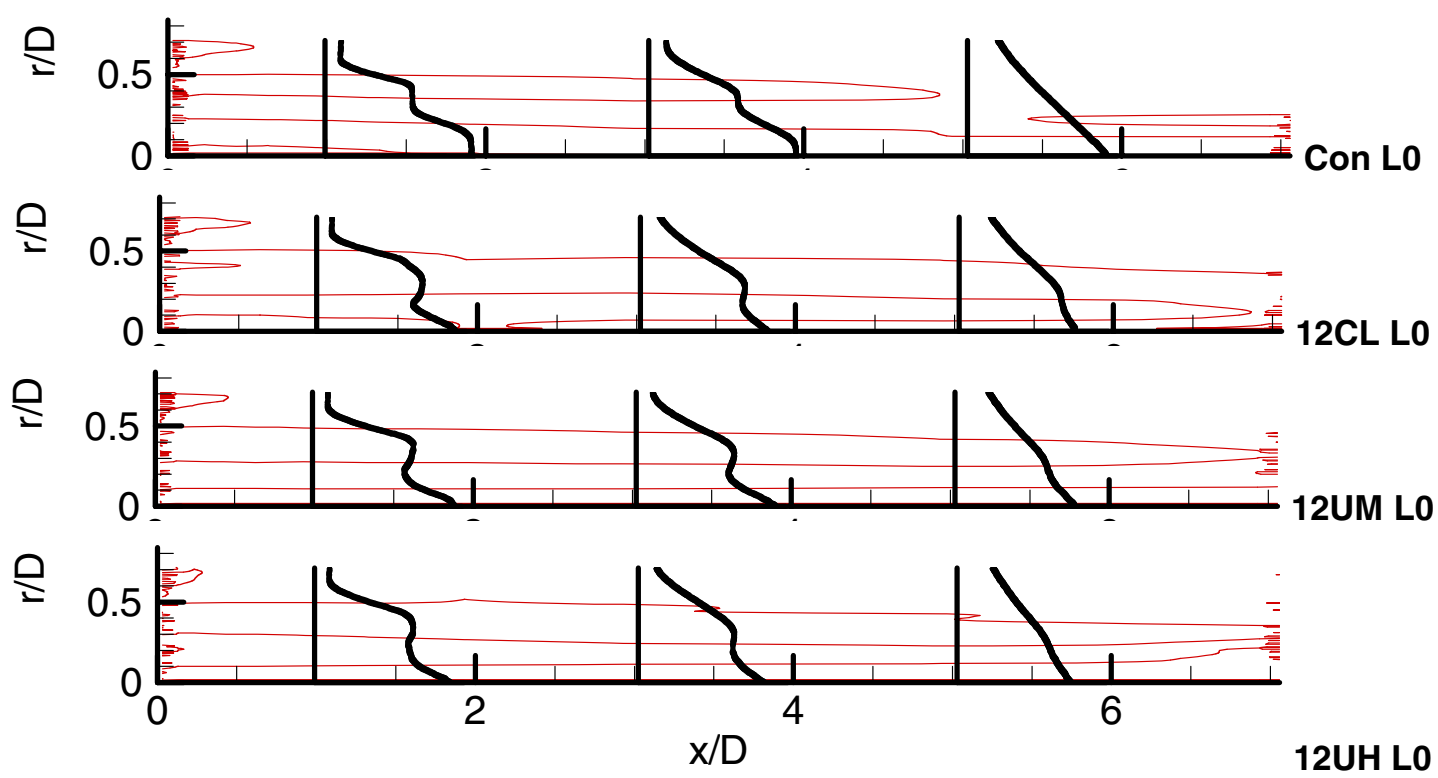

Figure 13.-Mapping inflection points in azimuthally averaged, mean velocity profiles of long nozzle L0 with low (12CL), medium (12UM), and high (12UH) penetration mixers and with axisymmetric (Conf) splitter. Setpoint 0312.

the velocities of inflection points in the mean velocity profile. In the current paper the convective Mach numbers of azimuthally averaged velocity fields are computed and checked for correlation with the aftdirected, low frequency noise of jets with different penetration mixers. The inflections points and representative mean velocity profiles are shown in figure 13 while the convective Mach numbers determined are plotted versus $\mathrm{x}$ in figure 14. In that figure the longer core of the confluent results in a substantially higher $\mathrm{M}_{\mathrm{c}}$ in the downstream regions of the jet. The analysis was too crude to establish differences in $\mathrm{M}_{\mathrm{c}}$ between mixers, but shows the promise of correlating the large-scale source with convective Mach number of the shear layers. 


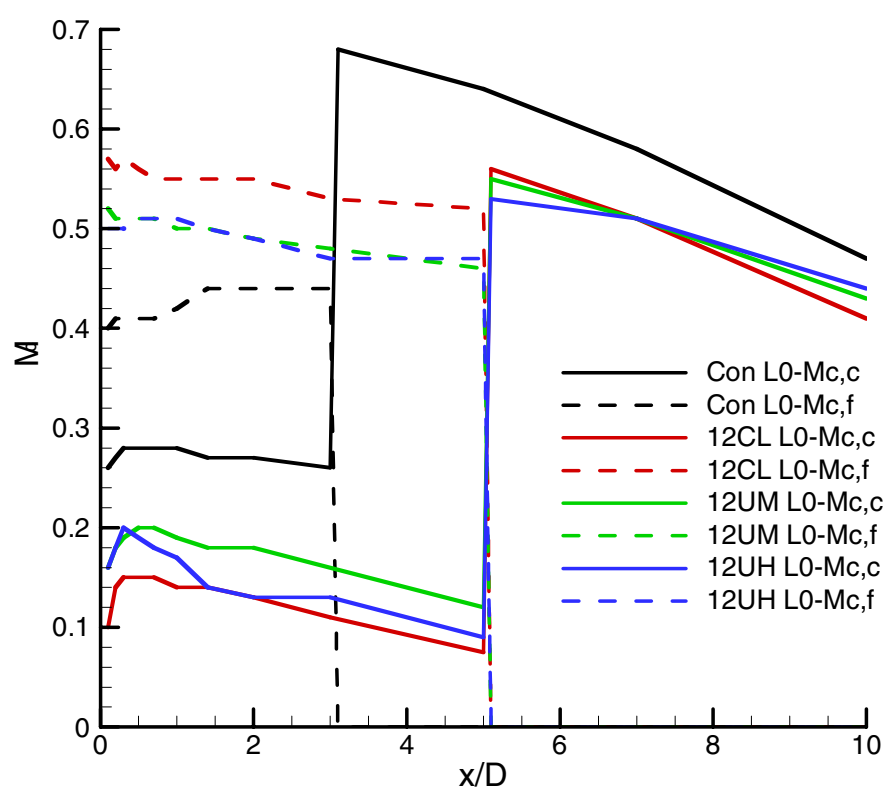

Figure 14.-Convective Mach numbers $\mathrm{M}_{\mathrm{c}}$ for shear layers of long nozzle L0 with low (12CL), medium (12UM), and high (12UH) penetration mixers and with axisymmetric

(Conf) splitter. Solid lines are core stream shear layer, dashed lines are fan stream shear layer.

\section{Summary}

Cross-stream PIV has been successfully used to measure the details of the turbulent flow fields downstream of internally mixed nozzles with lobed mixers. Three-component velocity fields were measured over the first 10 jet diameters focusing on the region near the jet exit. Several variations in mixers, nozzle length, flight speed, and cycle point were explored; results for various mixers have been highlighted in this presentation.

Comparisons with hotwire and LDV measurements were shown to ascertain the validity of the statistics extracted from the PIV data. Having vetted the data for accuracy, the flow field measurements were presented in ways amenable to source diagnostics and jet noise modeling efforts. As a diagnostic tool, the cross-stream PIV was used to confirm that high frequency sources associated with changes in nozzle length were not caused by flow features near the nozzle lip. For jet noise modeling, comparisons of the measured changes in turbulence and broadside noise fields with changes in mixer lobe penetration loosely confirmed results from acoustic analogy theory, but held a few intriguing contradictions for noise at aft angles. Here no significant differences were found in the turbulence corresponding to the strong acoustic differences between lobed mixers and axisymmetric splitters. Azimuthally averaged PIV measurements were rescaled to provide mean flow profiles suitable for equivalent jet modeling of the noise from hot dual-flow jets with lobed mixers, and analyzed for shear layer convective Mach numbers, two alternative methods of accounting for the acoustic differences in lobe geometry. 


\section{References}

1. Mengle,V.G., Baker, V.D., and Dalton, W.N., "Lobed mixer Design for Noise Suppression," NASA/CR-2002-210823, 2002.

2. Tester, B.J. and Fisher, M.J., "Understanding and prediction of jet noise generation by forced mixers," AIAA-2004-2897.

3. Kahler, C.J. and Kompenhans, J. "Multiple plane stereo PIV: technical realization and fluid mechanical significance", Proc. of the International Workshop on PIV, Santa Barbara, USA, 1999.

4. Hu, H. Saga, T., Kobayashi, T., Taniguchi, and N., Yasuki, M., "Dual-plane stereoscopic Particle Image Velocimetry system setup and its application on a lobed jet mixing flow," Expts. in Fluids Vol. 31, 277-293, 2001

5. Arakeri, V.H., Krothapalli, A., Siddavaram, V., Alkislar, M.B., and Lourenco, L.M., "On the use of microjets to suppress turbulence in a Mach 0.9 axisymmetric jet," J Fluid Mech. Vol. 490, 75-98, 2003

6. Wernet, M.P., "Stereo Viewing 3-Component, Planar PIV Utilizing Fuzzy Inference," Proceedings of the 19th AIAA conference on Advanced Measurement and Ground Testing Technology Conference, New Orleans, LA, June 17-20, 1996.

7. Wernet, M.P., "Fuzzy logic enhanced digital PIV processing software," 18th International Congress on Instrumentation in Aerospace Simulation Facilities (ICIASF), Toulouse, France, June, 1999.

8. Wernet, M.P., Skoch, G.J., and Wernet, J.H., "Demonstration of a Stabilized Alumina/Ethanol Colloidal Dispersion Technique for Seeding High Temperature Air Flows", 16th International Congress on Instrumentation for Aerospace Simulation Facilities (ICIASF), Wright-Patterson Air Force Base, July 18-21, 1995.

9. Bradshaw, P., Ferriss, D.H., and Johnson, R.F., "Turbulence in the noise-producing region of a circular jet," J. Fluid Mech., Vol 19, 591-624, (1964).

10. Ko, N.W.M., and Kwan, A.S.H., "The initial region of subsonic coaxial jets," J. Fluid Mech. Vol. 73, 305-322, (1976).

11. Gary Podboy, NASA Glenn Research Center, private communication.

12. Sang Soo Lee, NASA Glenn Research Center, private communication.

13. Fisher, M.J., Preston, G.A., \& Mead, C.J., 1998 "A modeling of the noise from simple coaxial jets, part II: with heated primary flow," J. Sound Vibration 209, 405-417.

14. Fisher, M.J., Preston, G.A., \& Bryce, W.D., 1998 "A modeling of the noise from simple coaxial jets, part I: with unheated primary flow," J. Sound Vibration 209, 395-403.

15. Papamoschou, D. "Convective Mach Wave attenuation with shaped profiles," AIAA Aerospace Sciences Meeting, Reno, NV 2004, AIAA Paper no. 2004-0004. 
Public reporting burden for this collection of information is estimated to average 1 hour per response, including the time for reviewing instructions, searching existing data sources, gathering and maintaining the data needed, and completing and reviewing the collection of information. Send comments regarding this burden estimate or any other aspect of this collection of information, including suggestions for reducing this burden, to Washington Headquarters Services, Directorate for Information Operations and Reports, 1215 Jefferson Davis Highway, Suite 1204, Arlington, VA 22202-4302, and to the Office of Management and Budget, Paperwork Reduction Project (0704-0188), Washington, DC 20503.

\begin{tabular}{|l|l|l}
\hline 1. AGENCY USE ONLY (Leave blank) & $\begin{array}{c}\text { 2. REPORT DATE } \\
\text { September } 2004\end{array}$ & $\begin{array}{r}\text { 3. REPORT TYPE AND DATES COVERED } \\
\text { Technical Memorandum }\end{array}$ \\
\hline
\end{tabular}

4. TITLE AND SUBTITLE

5. FUNDING NUMBERS

Cross-Stream PIV Measurements of Jets With Internal Lobed Mixers

6. AUTHOR(S)

WBS-22-781-30-24

James Bridges and Mark P. Wernet

7. PERFORMING ORGANIZATION NAME(S) AND ADDRESS(ES)

National Aeronautics and Space Administration

John H. Glenn Research Center at Lewis Field

Cleveland, Ohio 44135-3191

8. PERFORMING ORGANIZATION

REPORT NUMBER

E-14581

9. SPONSORING/MONITORING AGENCY NAME(S) AND ADDRESS(ES)

National Aeronautics and Space Administration

Washington, DC 20546-0001

10. SPONSORING/MONITORING

AGENCY REPORT NUMBER

NASA TM-2004-213106

AIAA-2004-2896

\section{SUPPLEMENTARY NOTES}

Prepared for the Tenth Aeroacoustics Conference cosponsored by the American Institute of Aeronautics and Astronautics and the Confederation of European Aerospace Societies, Manchester, United Kingdom, May 10-12, 2004. Responsible person, James Bridges, organization code 5940, 216-433-2693.

12a. DISTRIBUTION/AVAILABILITY STATEMENT

12b. DISTRIBUTION CODE

Unclassified - Unlimited

Subject Categories: 07 and 34

Distribution: Nonstandard

Available electronically at http://gltrs.grc.nasa.gov

This publication is available from the NASA Center for AeroSpace Information, 301-621-0390.

13. ABSTRACT (Maximum 200 words)

With emphasis being placed on enhanced mixing of jet plumes for noise reduction and on predictions of jet noise based upon turbulent kinetic energy, unsteady measurements of jet plumes are a very important part of jet noise studies. Given that hot flows are of most practical interest, optical techniques such as Particle Image Velocimetry (PIV) are applicable. When the flow has strong azimuthal features, such as those generated by chevrons or lobed mixers, traditional PIV, which aligns the measurement plane parallel to the dominant flow direction is very inefficient, requiring many planes of data to be acquired and stacked up to produce the desired flow cross-sections. This paper presents PIV data acquired in a plane normal to the jet axis, directly measuring the cross-stream gradients and features of an internally mixed nozzle operating at aircraft engine flow conditions. These nozzle systems included variations in lobed mixer penetration, lobe count, lobe scalloping, and nozzle length. Several cases validating the accuracy of the PIV data are examined along with examples of its use in answering questions about the jet noise generation processes in these nozzles. Of most interest is the relationship of low frequency aft-directed noise with turbulence kinetic energy and mean velocity.

14. SUBJECT TERMS

Jet flow; Jet exhaust; Flow distribution; Turbulent flow; Azimuth; Noise reduction; Scalloping;

Mixers; Acoustics; Turbulence; Plumes; Jet aircraft noise; Particle image velocimetry

17. SECURITY CLASSIFICATION OF REPORT

Unclassified

18. SECURITY CLASSIFICATION
OF THIS PAGE
Unclassified

Unclassified
19. SECURITY CLASSIFICATION OF ABSTRACT

Unclassified
15. NUMBER OF PAGES

16. PRICE CODE

20. LIMITATION OF ABSTRACT

Standard Form 298 (Rev. 2-89)

Prescribed by ANSI Std. Z39-18 298-102 

